\title{
Wellness e nefrolitiasi
}

\author{
Alberto Trinchieri \\ S.C. Urologia, Ospedale A. Manzoni, Lecco
}

\begin{abstract}
Wellness and nephrolithiasis
"Wellness" is a philosophy of life that has been increasingly successful in economically developed countries, as a response to lifestyles that have obvious negative consequences on the health of the population. It is based on a constant physical activity, a balanced diet and a positive mental approach. The adoption of this lifestyle could also have a positive impact on the prevention of kidney stones. According to the latest findings, a balancing of the content in lipids, proteins and carbohydrates of the diet with low intake of salt, saturated fat, simple carbohydrates (sugar) and cholesterol and an increased intake of fruits, vegetables and whole grains is highly desirable in renal stone patients. However the consumption of whole grains, vegetables and fruits should be modulated in order to avoid an excessive intake of oxalate and fructose. Regular and moderate exercise has the prerequisites for a positive effect in reducing the risk of kidney stone formation, although at the time the epidemiologic evidence of this positive effect remains weak. The capacity to adapt to stressful events of daily life could have a favorable effect on the risk of forming kidney stones as the amount of stressful events proved to be greater in patients with kidney stones.
\end{abstract}

Keywords: Diet, Physical activity, Stress, Urinary calculi, Wellness

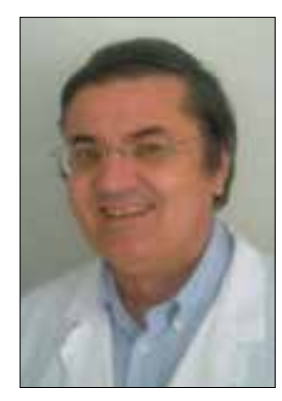

Alberto Trinchieri

\section{Introduzione}

Il vocabolo "wellness" è diventato di uso comune, anche nella lingua italiana. Secondo Wikipedia (1) è "una filosofia di vita che mette il benessere al centro dell'attenzione proponendo attività sportive e pratiche di rigenerazione oltre che di "mental training", combinate con un'alimentazione corretta".

La Wellness Foundation, una fondazione collegata con un'industria che produce attrezzature sportive, definisce il wellness come "lo stile di vita che, attraverso l'unione di attività fisica costante, sana alimentazione e approccio mentale positivo, permette alla persona di raggiungere il proprio equilibrio" (2).

Quale sia uno stile di vita "salutare" rimane, però, non ben definito, in quanto i principi che ispirano le norme co-

Accepted: November 17, 2015

Published online: January 20, 2016

Indirizzo per la corrispondenza:

Dr. Alberto Trinchieri

S.C. Urologia

Ospedale A. Manzoni

Via dell'Eremo 9/11

23900 Lecco

a.trinchieri@ospedale.lecco.it munemente suggerite per conseguire e mantenere un buono stato di salute non sono validati, se non parzialmente, da precise evidenze scientifiche.

Alcuni di questi principi si ispirano al concetto di "naturale", cioè al ripristino di abitudini caratteristiche della vita umana antecedentemente al processo di "civilizzazione". Altre convinzioni si basano sul "buon senso" o su antiche credenze popolari, mentre altre ancora su una diffusa sottocultura che viene veicolata dai mezzi di informazione tradizionale (riviste, giornali, radio, TV) o dalla rete.

Per alcune condizioni patologiche (malattie cardiovascolari, tumori, ecc.) esistono evidenze epidemiologiche, più o meno solide, che collegano alcuni stili di vita con l'insorgenza della specifica malattia.

Per la nefrolitiasi, l'impatto dello stile di vita sul rischio litogeno è stato solo episodicamente oggetto di osservazioni sistematiche, se si esclude lo studio dei fattori dietetici, che sono stati, invece, indagati in modo più estensivo.

Le componenti dello stile di vita che potrebbero essere prese in considerazione sono molteplici: dieta, attività fisica e sportiva, stress, fumo, alcool, esposizioni ambientali e così via.

\section{Dieta}

La maggior parte dei "website" che si occupano di "wellness" propugna una dieta "bilanciata", talvolta riferendosi a FAO/WHO, che hanno identificato, tra gli obiettivi dietetici di una popolazione sana, un apporto lipidico e un apporto proteico pari rispettivamente al $15-30 \%$ e al $10-15 \%$ del con- 
tenuto energetico totale, con il restante $55-75 \%$ di energia fornito dai carboidrati (3). Inoltre FAO/WHO suggeriscono un limitato apporto di acidi saturi (a vantaggio dei polinsaturi), di carboidrati semplici (zuccheri), di colesterolo e di sale e un aumentato apporto di frutta, verdura e cereali integrali. Nel loro documento sono stati proposti suggerimenti più specifici per la prevenzione del diabete, delle malattie cardiovascolari, dei tumori, delle malattie dentali e dell'osteoporosi, mentre la prevenzione della calcolosi renale non è stata presa in considerazione.

Tuttavia, quasi tutti gli obiettivi nutrizionali identificati per la promozione della salute in generale da FAO/WHO possono essere recepiti come efficaci anche per la prevenzione della nefrolitiasi.

La valutazione estensiva dei potenziali effetti della dieta sul rischio litogeno non è stata oggetto di questo studio. Una recente review (4) ha estensivamente esaminato i numerosi studi osservazionali di coorte e i pochi studi di intervento relativi al rischio dietetico di formazione di calcoli renali e alla possibilità di prevenire la calcolosi renale con opportuni regimi dietetici. Le risultanze di questa analisi confermano che un eccessivo apporto di proteine animali e sodio insieme a un insufficiente apporto di potassio e calcio e un'inadeguata idratazione sono i principali possibili fattori di rischio di formazione di calcoli urinari di calcio.

D'altra parte, il rischio litiasico si riduce con un'alimentazione aderente ai principi della dieta DASH (Dietary Approaches to Stop Hypertension), che recepisce molti principi del "wellness" (5).

Alcuni obiettivi della dieta "wellness" meritano, tuttavia, qualche ulteriore riflessione, in particolare il consumo di verdura e frutta e di cereali integrali, in relazione con l'apporto di ossalato e fruttosio.

Sebbene sia ben noto che l'ossalato è contenuto nei cibi di origine vegetale, le informazioni sul web in merito al contenuto di ossalato dei cibi sono molto contrastanti e spesso basate su informazioni datate o non affidabili. In un recente studio (6), sono stati esaminati sei website e 2 applicazioni per Smartphone, rilevando informazioni estremamente variabili sul contenuto di ossalato dei diversi alimenti. Tra i frut$\mathrm{ti}$, le oscillazioni maggiori sono state rilevate per le arance (2.07-10.6 mg/100 g) e le banane $(0-9.9 \mathrm{mg} / 100 \mathrm{~g})$, tra le verdure per gli spinaci (364.4-1145 mg/100 g), il rabarbaro (511$983.6 \mathrm{mg} / 100 \mathrm{~g})$ e le barbabietole $(36.9-794.1 \mathrm{mg} / 100 \mathrm{~g})$ e, tra la frutta secca, per le noccioline $(64.6-348.6 \mathrm{mg} / 100 \mathrm{~g}) \mathrm{e}$ le noci pecan (4.1-404.1 mg/100 g). Le ragioni della variabilità di queste informazioni sono molteplici. II metodo di dosaggio dell'ossalato nei cibi ha un ruolo fondamentale. Le prime misurazioni sono state eseguite con metodi colorimetrici ed enzimatici che si sono dimostrati, nel tempo, poco affidabili $(7,8)$. Più recentemente, hanno trovato impiego metodiche più affidabili, come l'elettroforesi capillare, la cromatografia ionica e la gascromatografia. L'elettroforesi capillare e la cromatografia ionica sono due metodi di indagine affidabili, che forniscono risultati comparabili, anche se la cromatografia ionica è più affidabile per la valutazione di piccole quantità di ossalato. La gascromatografia è un metodo altrettanto affidabile, ma richiede un passaggio preliminare per rendere l'ossalato volatile. La maggiore precisione di questi metodi ha consentito misure più attendibili e, per esempio, di dimostrare che le patate hanno un contenuto discretamente elevato di ossalato $(5.5-30 \mathrm{mg} / 100 \mathrm{~g})$, mentre, in precedenza, era stato stimato come molto basso (9). Tuttavia, anche con metodiche molto affidabili la stima del contenuto di ossalato rimane aleatoria, per la reale variabilità del contenuto di ossalato nei cibi freschi e conservati. Infatti, il contenuto di ossalato dipende dalla varietà della pianta, dallo stato di maturazione, dalla stagione e dalle condizioni di crescita (terreno, precipitazioni, ecc.). Un ulteriore fattore di variabilità è rappresentato dalla modalità di processazione degli alimenti e dalla modalità di preparazione dei cibi. Infine, l'ossalato presente negli alimenti non viene assorbito in modo costante, in quanto la biodisponibilità dell'ossalato durante i processi digestivi dipende dalla concentrazione di cationi chelanti l'ossalato come il calcio e il magnesio, dal tempo di transito nell'intestino tenue e crasso e dall'attività di degradazione batterica. Infine, esistono variazioni inter-individuali nell'assorbimento intestinale. II clinico e il divulgatore scientifico dovrebbero, quindi, tenere presente l'incertezza di alcune acquisizioni e moderare la perentorietà dei loro suggerimenti dietetici che, proscrivendo lunghi elenchi di alimenti, possono fuorviare l'attenzione del paziente dagli obiettivi principali dell'intervento dietetico. Per quanto riguarda l'ossalato, è, probabilmente, più vantaggioso richiedere di eliminare il consumo di un limitato numero di verdure che hanno un alto (o altissimo) contenuto di ossalato per porzione (Tab. I) (10). Dovrebbe essere considerato il contenuto per porzione, piuttosto che il contenuto per $100 \mathrm{~g}$, perché, per esempio, ha uno scarso significato proscrivere il consumo di prezzemolo, che ha un elevato contenuto di ossalato per $100 \mathrm{~g}$, ma che viene consumato in minime quantità!

È importante sottolineare che i cereali integrali, gli pseudocereali e le crusche hanno un elevato contenuto di ossalato, che è contenuto nello strato più esterno dei chicchi. Siener et al. hanno valutato accuratamente il contenuto di ossalato dei cereali integrali con HPLC a reattore enzimatico, una metodica sofisticata per l'identificazione dell'ossalato in matrici complesse (11). Il contenuto di ossalato si è dimostrato elevato $(>50 \mathrm{mg} / 100 \mathrm{~g}$ ) in alcuni cereali integrali come il grano duro (Triticum durum) $(76.6 \mathrm{mg} / 100 \mathrm{~g})$ e il grano tenero (Triticum aestivum) $(53.3 \mathrm{mg} / 100 \mathrm{~g})$, che sono i componenti rispettivamente della pasta (ma anche del cuscus e del bulgur), della farina e dei fiocchi di frumento. La crusca di frumento presenta i più alti contenuti di ossalato (457.4 $\mathrm{mg} / 100 \mathrm{~g}$ ). D'altra parte, già con metodiche più tradizionali era stato identificato l'elevato contenuto di ossalato della crusca di frumento (12). La somministrazione di crusca di grano è stata presa in considerazione in passato per la prevenzione 
TABELLA I - Alimenti con alto/altissimo contenuto di ossalato (nutrition department of Harvard school of public health) (10)

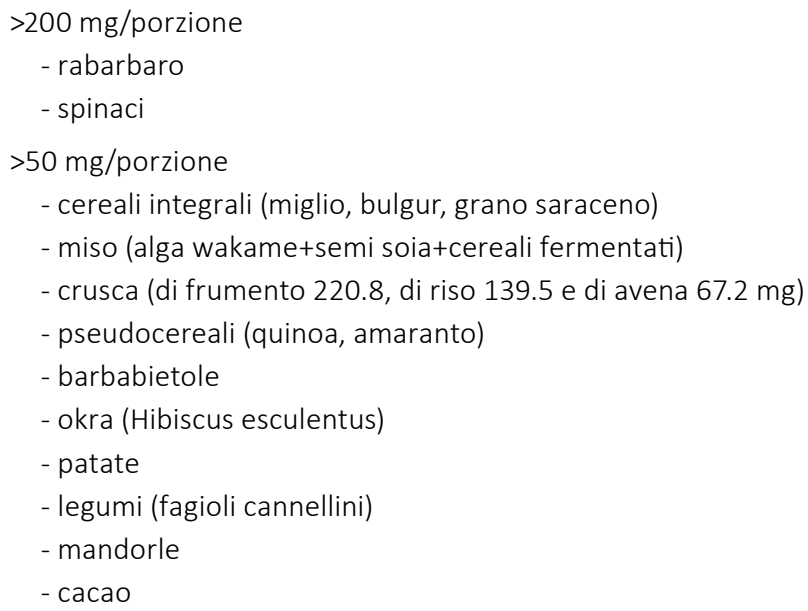

della calcolosi renale, in quanto è efficace nel ridurre l'assorbimento intestinale di calcio e la calciuria, ma il suo impiego è stato presto abbandonato, in quanto causa secondariamente un incremento dell'ossalato urinario (13).

La somministrazione di $36 \mathrm{~g}$ al giorno diversi tipi di crusca (riso, soia e frumento) ha ridotto l'escrezione urinaria di calcio, ma ha incrementato l'escrezione di ossalato, con un effetto complessivo sfavorevole sulla saturazione urinaria per l'ossalato di calcio (14).

In uno studio non controllato, la somministrazione di crusca di riso ha ridotto la frequenza di formazione dei calcoli renali rispetto al periodo antecedente lo studio, sebbene fosse confermato l'effetto sfavorevole sull'escrezione di ossalato (15).

Uno dei pochi studi randomizzati di intervento dietetico per la prevenzione della calcolosi renale prevedeva un complesso regime dietetico che comprendeva una riduzione dell'apporto proteico e un maggiore apporto di frutta e verdura, associati a un supplemento giornaliero di crusca di frumento (1/4 di tazza) (16). Lo studio ha sorprendentemente concluso che questo regime dietetico non è vantaggioso per la prevenzione della calcolosi renale rispetto al semplice incremento dell'apporto idrico. Il ruolo del supplemento di crusca deve essere attentamente considerato nella valutazione dell'insuccesso di questo studio.

In conclusione, il frequente consumo di cereali integrali e l'assunzione di crusca devono essere considerati attentamente nei pazienti formatori di calcoli renali e devono essere, eventualmente, sconsigliati, soprattutto se si associano a livelli border-line di ossaluria.

Infine, deve essere considerato il ruolo del fruttosio come altro possibile fattore di rischio per la calcolosi urinaria.

Il consumo eccessivo di fruttosio può aumentare l'escrezione urinaria di calcio, ossalato e acido urico e, conseguentemente, il rischio di formazione dei calcoli renali (17-19).

Lo studio di Taylor e Curhan ha dimostrato, in tre nume- rose popolazioni, che il rischio di formare un calcolo renale è stato superiore nei soggetti con apporto dietetico di fruttosio più elevato, mentre il consumo di altri carboidrati diversi dal fruttosio non ha influenzato il rischio di calcolosi (20).

Il consumo di fruttosio è aumentato in modo considerevole negli ultimi decenni negli Stati Uniti, in parallelo al consumo dello sciroppo di acero.

II fruttosio può essere consumato come fruttosio libero (monosaccaride) o come derivato dal saccarosio, cioè un disaccaride composto da glucosio e fruttosio.

Le fonti più comuni di fruttosio libero sono le bibite con aggiunta di zuccheri, le mele, le banane, il succo di arancia o mela e l'uva; quelle di saccarosio sono lo zucchero da tavola, le bibite addizionate di zuccheri, i succhi di frutta, i dolci, i gelati e la frutta sciroppata.

Conseguentemente, bisogna incoraggiare i pazienti con calcolosi urinaria a consumare preferenzialmente la verdura rispetto alla frutta e, comunque, a consumare con cautela i frutti a più alto contenuto di fruttosio (mele, banane, uva).

\section{Alcool}

II consumo moderato di bevande alcoliche non è associato a un aumento del rischio di calcolosi, ma, viceversa, sembra avere un effetto protettivo. Già negli anni '80, Shuster et al. avevano dimostrato che il consumo di bibite addizionate con anidride carbonica era associato a un maggiore rischio di calcolosi renale, mentre il consumo di birra e vino era associato a una diminuzione del rischio (21). Questa osservazione è stata confermata, in seguito, da numerosi studi. Curhan et al., in una coorte di oltre 45000 uomini di età tra i 40 e i 75 anni, seguiti per un periodo di 6 anni, hanno osservato una riduzione del rischio di calcolosi renale del $10 \%$ per il consumo di $250 \mathrm{cc} /$ die di caffè (decaffeinato o meno), del 14\% per $250 \mathrm{cc} /$ die di tè, del $21 \%$ e del $39 \%$ per ogni $250 \mathrm{cc} /$ die di birra e vino (22). Un gruppo di Autori finlandesi ha studiato una coorte di fumatori di sesso maschile di età tra i 50 e i 69 anni, nell'ambito dello studio Alpha-Tocopherol, Beta-Carotene Lung Cancer Prevention Study, che avevano risposto a un questionario dietologico all'inizio di un'indagine prospettica. Nel follow-up di 5 anni è stata valutata l'incidenza di calcoli renali, dimostrando che questa era inversamente associata al consumo di bir$\mathrm{ra}$, con una riduzione del rischio del $40 \%$ per ogni bottiglia di birra (23). Infine, Ferraro et al., in uno studio prospettico su 3 coorti numerose di soggetti, hanno valutato l'associazione tra il consumo di alcune bevande e il rischio di calcolosi renale, dimostrando che il rischio è aumentato del $25 \%$ tra coloro che fanno frequente consumo di cola zuccherata, del 33\% per altre bevande zuccherate non-cola e del $18 \%$ tra i consumatori di punch, mentre è ridotto del $26 \%$ e del $16 \%$ tra i frequenti consumatori di caffè o caffè decaffeinato e del 31-33\% e del $41 \%$ tra i consumatori di vino e birra (24). Una recente metanalisi, che ha considerato i risultati di 15 studi (10 studi di coorte e 5 caso-controllo), ha dimostrato un effetto protettivo 
sul rischio di formazione di calcoli renali legato al consumo di alcool $(\mathrm{RR}=0.80,95 \% \mathrm{Cl}: 0.75,0.85 ; \mathrm{P}<0.01) \mathrm{e}$, anche se in minor misura, al consumo di caffè (25).

\section{Attività fisica}

L'esercizio fisico implica una riduzione del rischio per molte patologie, come le malattie cardiovascolari, l'ipertensione, l'ictus, il diabete tipo 2, l'osteoporosi, l'obesità, alcune neoplasie e le sindromi ansioso-depressive. In particolare, l'attività fisica ha un effetto benefico nel prevenire alcune malattie croniche che possono associarsi alla nefrolitiasi, come l'ipertensione arteriosa, l'osteoporosi e il diabete mellito (26-28).

Inoltre, l'attività fisica potrebbe avere un effetto protettivo specifico sul rischio di formazione di calcoli renali. La sete conseguente all'esercizio fisico può promuovere l'assunzione di liquidi anche in eccesso rispetto alle possibili perdite attraverso la sudorazione, con conseguente incremento dell'acqua corporea totale (29). L'escrezione urinaria di sodio si riduce per effetto della perdita cutanea attraverso la sudorazione, con conseguente aumentato riassorbimento tubulare (30). II volume ematico circolante si espande del $20-25 \%$, con conseguente ridotta stimolazione del sistema nervoso simpatico $(31,32)$. Inoltre l'aumento del carico sul sistema osteo-articolare ha un effetto favorevole sul contenuto minerale osseo e riduce il rischio di osteoporosi (33-35). Viceversa, i soggetti che sono inattivi fisicamente tendono ad avere una contrazione del volume circolante, con aumento della viscosità ematica e stimolazione del sistema nervoso simpatico e un incremento dei livelli ematici di colesterolo LDL (36). L'attività fisica, anche se moderata o settimanale, contrasta l'insulino-resistenza, riduce l'obesità viscerale addominale e migliora il profilo lipidico.

Sorensen et al. (37) hanno valutato i dati relativi a circa 84000 donne in postmenopausa seguite in modo prospettico dal 1993 nell'ambito del Women's Health Initiative Observations Study. In questa coorte, le pazienti con indice di massa corporea più elevato presentavano un aumentato rischio di formazione di calcoli renali; inoltre, un maggiore apporto calorico e una ridotta attività fisica hanno dimostrato di essere fattori di rischio indipendenti per la formazione di calcoli renali. La sedentarietà potrebbe, quindi, rappresentare un fattore di rischio per la calcolosi renale, sebbene l'intensità dell'attività fisica non sembri avere particolare importanza perché l'effetto protettivo si raggiunge anche con una modesto livello di attività fisica. L'effetto protettivo si è manifestato oltre una soglia di $10 \mathrm{METs} /$ settimana, che equivale a 3-4 ore di camminata spedita (3-5 km/ora) o a 4 ore di giardinaggio o a 1 ora di jogging (9-10 km/ora) (Tab. II) (38). In uno studio successivo (39), in tre numerose coorti di soggetti studiate prospetticamente, Ferraro et al. hanno osservato una correlazione inversa tra livello di attività fisica e rischio di formazione di calcoli renali solo in due delle popolazioni studiate (NHS I e NHS II), che, tuttavia, non è stata confermata dopo l'aggiustamento per altre co-variabili. Analogamente, gli Autori non
TABELLA II - Consumo energetico di diverse attività fisiche

\begin{tabular}{lc}
\hline Attività & METs/ora \\
\hline $\begin{array}{l}\text { Marcia lenta (meno } 3 \mathrm{~km} / \mathrm{h} \text { ) - Giardinaggio } \\
\text { leggero - Attività domestiche }\end{array}$ & $2.0-2.3$ \\
Marcia veloce $(5 \mathrm{~km} / \mathrm{h}$ ) - Giardinaggio pesante - & $3.3-4.0$ \\
Marcia in salita - Ciclismo (meno di $15 \mathrm{~km} / \mathrm{h})$ & \\
Danza - Snorkeling & $4.8-5.0$ \\
Tagliare erba con tagliaerba a mano - Spalare neve & $5.5-6.0$ \\
Canottaggio - Sci in discesa & $6.0-8.0$ \\
Ciclismo (25 km/h) - Ginnastica a corpo libero & $8.0-10.0$ \\
intensa - Tennis (singolo) - Nuoto & \\
Corsa (12 km/h) & 13.5 \\
\hline
\end{tabular}

hanno potuto confermare un'associazione tra apporto calorico totale e rischio di formazione di calcoli renali. La differenza di questi risultati può essere spiegata dal fatto che lo studio di Sorensen era limitato a donne in post-menopausa (Women's Health Initiative, WHI), mentre le popolazioni osservate da Ferraro erano relative a entrambi i sessi. Inoltre, le abitudini delle popolazioni studiate da Ferraro erano più "salutistiche" rispetto a quelle dello studio di Sorensen, in quanto il numero di soggetti con livelli di attività fisica molto ridotta (<0.1 METs/settimana) era esiguo, per cui il confronto con una popolazione "inattiva" non è stato possibile. Infine, è interessante osservare che Nouvenne et al. (40) hanno osservato che la diffusione di una cultura "salutistica", in parte mediata da Internet, ha modificato, nel corso degli ultimi 25 anni, le abitudini dei pazienti formatori di calcoli renali in Italia: l'apporto idrico è aumentato da 1.37 a $1.78 \mathrm{~L} /$ die negli uomini e da 1.21 a $1.55 \mathrm{~L} /$ die nelle donne e la percentuale dei pazienti che sono fisicamente attivi è passata dall' $8 \%$ al $41 \%$.

\section{Fumo}

Come è ben noto, il tabagismo è causa di effetti nocivi sulla salute, in quanto favorisce l'insorgenza di neoplasie, malattie dell'apparato respiratorio e malattie cardiovascolari. Le sostanze tossiche o cancerogene presenti nel fumo di sigaretta sono numerose: nicotina, monossido di carbonio, benzene e benzopireni, polveri fini e sostanze irritanti. II fumo di sigaretta espone, inoltre, all'inalazione di cadmio nella quantità di circa il $10 \%$ dei 1-2 microgrammi presenti in ogni sigaretta; ciò può aumentare i livelli di intake totale, in particolare nei soggetti già esposti professionalmente (41). Alcuni studi sperimentali nell'animale hanno dimostrato che la nicotina riduce la peristalsi ureterale (42), probabilmente attraverso la stimolazione dei recettori colinergici presenti sull'uretere (43). Conseguentemente, il fumo di sigaretta potrebbe avere un effetto sfavorevole sull'espulsione spontanea dei calcoli ureterali a causa degli elevati livelli ematici di nicotina conseguenti al passaggio nel circolo ematico di circa il $50 \%$ della nicotina presente nel fumo inspirato (44). Un recente studio (45) non ha, tuttavia, dimostrato alcun effetto significativo sull'espulsione spontanea di calcoli dell'uretere di- 
stale, sebbene la percentuale di espulsione spontanea dei calcoli $>4 \mathrm{~mm}$ tenda a essere inferiore nei fumatori. Per quanto riguarda il cadmio, è stato dimostrato che l'esposizione a livelli moderatamente elevati di cadmio ( $>1 \mu \mathrm{g} / \mathrm{g}$ ) implica un maggiore rischio di formazione di calcoli renali, soprattutto nel sesso femminile (46). D'altra parte, lo studio degli elementi traccia nelle urine e nei calcoli di pazienti formatori di calcoli non ha dimostrato differenze significative tra fumatori e non fumatori (47). In conclusione, gli elementi per poter considerare il fumo di sigaretta tra i fattori di rischio per la calcolosi renale sono molto labili. Tuttavia, esistono precise evidenze di una correlazione tra osteoporosi, espressa dal rischio complessivo di fratture, e fumo (48). I meccanismi patogenetici implicati nell'effetto del fumo sull'osso sono complessi (ormonali, riduzione della massa corporea, riduzione dei livelli di vitamina $D$, stress ossidativo, vasculopatia periferica, ridotta performance neuromuscolare, effetto tossico diretto), ma l'ipotesi di un possibile effetto del fumo sul rischio litogeno meriterebbe di essere verificata con ulteriori studi epidemiologici.

\section{Stress psichico}

Agli inizi degli anni '80, il possibile ruolo dello stress psichico nella patogenesi della nefrolitiasi è stato oggetto di numerosi studi da parte degli Autori di lingua tedesca. Schneider et al. hanno rilevato fattori anamnestici suggestivi di stress psicologico nel $25 \%$ di una popolazione di pazienti con calcolosi renale (49). La maggior parte di questi pazienti lamentava un sovraccarico di attività lavorativa con scarsa possibilità di recupero psicofisico durante il week-end. Questa condizione implicava uno stato nevrotico, più marcato nel sesso femminile. Gli eventi stressanti aumenterebbero l'escrezione urinaria di soluti litogeni (calcio, ossalato e acido urico) e ridurrebbero l'escrezione di inibitori della cristallizzazione (magnesio e citra- to) $(50,51)$. In particolare, il rapporto tra calcio e magnesio nelle urine tenderebbe a ridursi (52). Tuttavia, il disegno di questi primi studi e la ridotta numerosità delle casistiche limitano il significato di queste osservazioni. L'effetto di una condizione di stress è stato studiato nell'animale (ratto) sottoposto a deboli stimoli elettrici ripetitivi (53). Questa condizione si associava a un'ipertrofia delle ghiandole surrenaliche. L'escrezione urinaria di calcio e sodio rimaneva immodificata, mentre si osservavano una riduzione dell'escrezione di potassio e magnesio e un aumento dell'escrezione di fosfato e acido urico. Le variazioni della composizione delle urine in risposta allo stress sono state spiegate come conseguenza della risposta di "attacco o fuga" a eventi stressanti, che implica la stimolazione delle cellule neurosecretorie dei nuclei soprattico e paraventricolare dell' ipotalamo con conseguente risposta ipofisaria. La secrezione di vasopressina riduce il volume urinario e provoca ipertonicità urinaria (54). Alcuni studi epidemiologici più recenti hanno riesaminato la possibile relazione tra stress e rischio di calcolosi renale (55). Najem et al. hanno confrontato, in uno studio caso-controllo, 200 pazienti con calcolosi renale con 200 controlli rispetto alla pregressa esposizione a eventi percepiti come molto stressanti, con un intenso impatto emotivo e apprensione per la durata di almeno una settimana (56). L'evenienza di eventi stressanti si è dimostrata significativamente maggiore nei pazienti rispetto ai controlli, giustificando l'ipotesi che possa esistere una correlazione tra stress emotivo e formazione di calcoli renali. L'analisi logistica multivariata ha identificato, tra le condizioni di rischio più significative, una condizione di preoccupazione per il reddito familiare e per la sostenibilità di un mutuo finanziario. In un altro studio caso controllo, sono stati confrontati 97 pazienti con 97 controlli appaiati per sesso ed età (57). Gli eventi stressanti sono stati misurati in base alla Social Readjustment Rating Scale (Tab. III) (58), il cui punteggio è stato significativamente più alto nei pazienti che nei control-

TABELLA III - Alcune cause di stress e relative classi di punteggio secondo la Social Readjustment Rating Scale (SRRS) (modificato da Holmes and Rahe Stress Scale) (58)

\begin{tabular}{l}
\hline Stress coniugale \\
Morte del coniuge \\
Divorzio - Separazione \\
Matrimonio - Riconciliazione coniugale - Aumento litigiosità coniugale - Inizio/fine attività lavorativa coniuge \\
Stress familiare e personale \\
Morte di un parente prossimo \\
Problemi di salute di un familiare - Gravidanza - Disfunzione sessuale - Nascita di un nuovo membro della famiglia - Morte \\
di un amico - Mutuo sulla casa $>100.000$ euro - Ottenimento per ristrutturazione di mutuo/prestito - Uscita di casa di \\
un(a) figlio(a) - Conseguimento di risultati personali rilevanti - Inizio/fine ciclo scolastico \\
Variazione delle condizioni di vita - Variazione delle abitudini personali - Cambio di residenza - Cambio di scuola - \\
Variazione delle abitudini sociali/ricreative - Prestito o mutuo $<100.000$ euro - Variazione del ritmo del sonno - Variazione \\
della frequenza delle riunioni familiari - Variazione delle abitudini alimentari \\
Stress sociale/lavorativo \\
Carcere \\
Licenziamento - Pensionamento - Riorganizzazione dell'attività commerciale - Riorganizzazione finanziaria - Cambio \\
mansione lavorativa - Aumento delle responsabilità lavorative - Problemi giudiziari \\
Problemi con il capoufficio/proprietario - Cambio condizioni lavorative \\
Un punteggio complessivo superiore a 300 indica un grave rischio patologico, tra 150 e 300 un rischio moderato e \\
inferiore a 150 un rischio lieve \\
\hline
\end{tabular}


TABELLA IV - Perceived stress scale scoring (scala per lo stress percepito) $(60,61)$
Il punteggio si basa su 10 domande che indagano l'ultimo mese.
1) Perdita di controllo rispetto a un evento imprevisto
2) Sensazione di mancanza di controllo della propria vita
3) Sensazione di essere nervoso/stressato
4) Sensazione di capacità di gestire i problemi personali
5) Sensazione di buon andamento degli eventi esistenziali
6) Sensazione di non riuscire a provvedere alle proprie attività
7) Capacità di gestione degli eventi irritanti
8) Capacità di padroneggiare la situazione
9) Frequenza di episodi di irritazione per incapacità a gestire eventi fuori controllo
10) Sensazione di accumulo di eventi non gestibili

Ogni domanda prevede 4 risposte $(0=$ Mai; 1 = Quasi mai; $2=$ A volte; $3=$ Abbastanza spesso; 4 = Molto spesso) che formano un punteggio da 0 a 4 .

li. L'analisi di regressione logistica ha dimostrato che il rischio di eventi stressanti con punteggio $>100$ è 3.02 volte più frequente nei pazienti con calcolosi e che il rischio di eventi molto stressanti con punteggio >200 è 2.87 volte maggiore che nei controlli. Miyaoka et al. (59) hanno studiato, con un questionario autosomministrato, 200 pazienti con calcolosi renale per misurare lo stress emotivo mediante il punteggio Perceived Stress Scale-10 [PSS-10] (Tab. IV) $(60,61)$. In questa popolazione, la condizione di stress emotivo si è dimostrata più frequente nel sesso femminile, in caso di una condizione di disoccupazione lavorativa, e nei pazienti con sintomatologia dolorosa in atto al momento dell'intervista e in quelli con una frequenza di 2 o più calcoli/anno.

\section{Conclusioni}

Il rapporto tra "wellness" e rischio di calcolosi renale non è ben definito. Alcuni principi dietetici che si associano al concetto di "wellness" possono avere un effetto protettivo sul rischio di litogenesi, ma il consumo, in modo esagerato e ripetitivo, di altri alimenti considerati "salutari" può avere conseguenze negative in termini di prevenzione della calcolosi renale. L'impatto dell'attività fisica "moderata" sembrerebbe essere positivo, mentre, in caso di un'attività intensa, deve essere esercitata molta attenzione nel ripristino dell'equilibrio idro-elettrolitico attraverso l'assunzione adeguata di liquidi. La capacità di prevenire e gestire eventi stressanti ha un ruolo positivo. II clinico, attento ai problemi della calcolosi urinaria, dovrebbe includere nello studio dei suoi pazienti un'adeguata informazione anche in merito all'attività fisica (lavorativa e ricreazionale) e al possibile ruolo di recenti o continui eventi stressanti. Tra i consigli terapeutici, il clinico e il divulgatore dovrebbero inserire raccomandazioni a favore di un'attività fisica costante e moderata e dell'adozione di tecniche di rilassamento mentale.

\section{Disclosures}

Financial support: No financial support was received for this submission. Conflict of interest: The author has no conflict of interest.

\section{Bibliografia}

1. https://it.wikipedia.org/wiki/

2. http://www.wellnessfoundation.it/

3. World Health Organization, Food and Agriculture Organization of the United Nations. Diet, nutrition and the prevention of chronic diseases Report of a joint WHO/FAO expert consultation. WHO Technical Report Series 916,2003.

4. Prezioso D, Strazzullo $\mathrm{P}$, Lotti $\mathrm{T}$, et al. Dietary treatment of urinary risk factors for renal stone formation. A review of CLU Working Group. Arch Ital Urol Androl 2015;87:105-20.

5. Taylor EN, Fung TT, Curhan GC. DASH-style diet associated with reduced risk for kidney stones. J Am Soc Nephrol 2009;20:2253-9.

6. Attalla K, De S, Monga M. Oxalate content of food: a tangled web. Urology 2014;84:555-60.

7. Hodgkinson A, Williams A. An improved colorimetric procedure for urine oxalate. Clin Chim Acta 1972;36:127-32.

8. Kasidas GP, Rose GA. Oxalate content of some common foods: determination by an enzymatic method. J Hum Nutr 1980;34:255-66.

9. Holmes RP, Kennedy M. Estimation of the oxalate content of foods and daily oxalate intake. Kidney Int 2000;57:1662-7.

10. https://regepi.bwh.harvard.edu/health/Oxalate/files

11. Siener R, Hönow R, Voss S, Seidler A, Hesse A. Oxalate content of cereals and cereal products. J Agric Food Chem 2006;54:3008-11.

12. Massey LK, Roman-Smith H, Sutton RA. Effect of dietary oxalate and calcium on urinary oxalate and risk of formation of calcium oxalate kidney stones. J Am Diet Assoc 1993;93:901-6.

13. Gleeson MJ, Thompson AS, Mehta S, Griffith DP. Effect of unprocessed wheat bran on calciuria and oxaluria in patients with urolithiasis. Urology 1990;35:231-4.

14. Jahnen A, Heynck H, Gertz B, Classen A, Hesse A. Dietary fibre: the effectiveness of a high bran intake in reducing renal calcium excretion. Urol Res 1992;20:3-6.

15. Ebisuno S, Morimoto S, Yasukawa S, Ohkawa T. Results of longterm rice bran treatment on stone recurrence in hypercalciuric patients. Br J Urol 1991;67:237-40.

16. Hiatt RA, Ettinger B, Caan B, Quesenberry CP Jr, Duncan D, Citron JT. Randomized controlled trial of a low animal protein, high fiber diet in the prevention of recurrent calcium oxalate kidney stones. Am J Epidemiol 1996;144:25-33.

17. Milne DB, Nielsen FH. The interaction between dietary fructose and magnesium adversely affects macromineral homeostasis in men. J Am Coll Nutr 2000;19:31-7.

18. Nguyen NU, Dumoulin G, Henriet MT, et al. Increase in urinary calcium and oxalate after fructose infusion. Horm Metab Res 1995;27:155-8.

19. Emmerson BT. Effect of oral fructose on urate production. Ann Rheum Dis 1974;33:276-80.

20. Taylor EN, Curhan GC. Fructose consumption and the risk of kidney stones. Kidney Int 2008;73:207-12.

21. Shuster J, Finlayson B, Scheaffer RL, Sierakowski R, Zoltek J, Dzegede S. Primary liquid intake and urinary stone disease. J Chronic Dis 1985;38:907-14.

22. Curhan GC, Willett WC, Rimm EB, Spiegelman D, Stampfer MJ. Prospective study of beverage use and the risk of kidney stones. Am J Epidemiol 1996;143:240-7.

23. Hirvonen T, Pietinen $P$, Virtanen $M$, Albanes D, Virtamo J. Nutrient intake and use of beverages and the risk of kidney stones among male smokers. Am J Epidemiol 1999;15;150:187-94.

24. Ferraro PM, Taylor EN, Gambaro G, Curhan GC. Soda and other 
beverages and the risk of kidney stones. Clin J Am Soc Nephrol 2013;8:1389-95.

25. $\mathrm{Xu} \mathrm{C}$, Zhang $\mathrm{C}$, Wang $\mathrm{XL}$, et al. Self-Fluid Management in Prevention of Kidney Stones: A PRISMA-Compliant Systematic Review and Dose-Response Meta-Analysis of Observational Studies. Medicine 2015;94:e1042.

26. Nelson ME, Rejeski WJ, Blair SN, et al. Physical activity and public health in older adults: Recommendation from the American College of Sports Medicine and the American Heart Association. Circulation 2007;116:1094-105.

27. Orwoll ES, Bauer DC, Vogt TM, Fox KM. Axial bone mass in older women. Study of Osteoporotic Fractures Research Group. Ann Intern Med 1996;124:187-96.

28. Reis JP, Loria CM, Sorlie PD, Park Y, Hollenbeck A, Schatzkin A. Lifestyle factors and risk for new-onset diabetes: A populationbased cohort study. Ann Intern Med 2011;155:292-9.

29. Convertino VA. Blood volume: Its adaptation to endurance training. Med Sci Sports Exerc 1991;23:1338-48.

30. Wade CE. Response, regulation, and actions of vasopressin during exercise: A review. Med Sci Sports Exerc 1984;16:506-11.

31. Brotherhood J, Brozovic B, Pugh LG. Haematological status of middle and long-distance runners. Clin Sci Mol Med 1975;48: 139-45.

32. Dill DB, Braithwaite K, Adams WC, Bernauer EM. Blood volume of middle-distance runners: Effect of 2,300-m altitude and comparison with non-athletes. Med Sci Sports 1974;6:1-7.

33. Pocock N, Eisman J, Gwinn T, et al. Muscle strength, physical fitness, and weight but not age predict femoral neck bone mass. J Bone Miner Res 1989;4:441-8.

34. Nelson ME, Fiatarone MA, Morganti CM, Trice I, Greenberg RA, Evans WJ. Effects of high-intensity strength training on multiple risk factors for osteoporotic fractures. A randomized controlled trial. JAMA 1994;272:1909-14.

35. Vezzoli G, Soldati L, Arcidiacono T, et al. Urinary calcium is a determinant of bone mineral density in elderly men participating in the In CHIANTI study. Kidney Int 2005;67:2006-14.

36. Stevenson ET, Davy KP, Seals DR. Maximal aerobic capacity and total blood volume in highly trained middle-aged and older female endurance athletes. J Appl Physiol 1994;77:1691-6.

37. Sorensen MD, Chi T, Shara NM, et al. Activity, energy intake, obesity, and the risk of incident kidney stones in postmenopausal women: a report from the Women's Health Initiative. J Am Soc Nephrol 2014;25:362-9.

38. Ainsworth BE, Haskell WL, Herrmann SD, et al. 2011 Compendium of Physical Activities: a second update of codes and MET values. Med Sci Sports Exerc 2011;43:1575-81.

39. Ferraro PM, Curhan GC, Sorensen MD, Gambaro G, Taylor EN. Physical activity, energy intake and the risk of incident kidney stones. J Urol 2015;193:864-8.

40. Nouvenne A, Ticinesi A, Allegri F, et al. Twenty-five years of idiopathic calcium nephrolithiasis: has anything changed? Clin Chem Lab Med 2014;52:337-44.

41. World Health Organization (WHO) Environmental Health Criteria 134. Cadmium International Programme on Chemical Safety (IPCS) Monograph, 1992.

42. Boyarsky S, Labay P, Pfautz CJ. The effect of nicotine upon ureteral peristalsis. South Med J 1968;61:573-9.

43. Schulman CC, Duarte-Escalante O, Boyarsky S. The ureterovesical innervation. A new concept based on a histochemical study. Br J Urol 1972;44(6):698-712.

44. Isaac PF, Rand MJ. Blood levels of nicotine and physiological effects after inhalation of tobacco smoke. Eur J Pharmacol 1969;8(3):269-83.

45. Fazlioglu A, Salman Y, Tandogdu Z, Kurtulus FO, Bas S, Cek $M$. The effect of smoking on spontaneous passage of distal ureteral stones. BMC Urol 2014;14:27.

46. Ferraro PM, Bonello M, Frigo AC, D’Addessi A, Sturniolo A, Gambaro G. Cadmium exposure and kidney stone formation in the general population--an analysis of the National Health and Nutrition Examination Survey III data. J Endourol 2011;25: 875-80.

47. Słojewski M, Czerny B, Safranow K, et al. Does smoking have any effect on urinary stone composition and the distribution of trace elements in urine and stones? Urol Res 2009;37: 317-22.

48. Kanis JA, Johnell O, Oden A, et al. Smoking and fracture risk: a meta-analysis. Osteoporos Int 2005;16:155-62.

49. Schneider HJ, Schüler G, Janitzky H. [Psychosomatic parameters in urolithiasis and their relation to sex and type of calculus-study using a modified complaint questionnaire]. Z Urol Nephrol 1980;73:523-30.

50. Brundig P, Berg W, Schneider HJ. [Investigations on formation risk of calcium oxalate stones with special regard to stress]. Urol Int 1979;34:105-13.

51. Brundig $\mathrm{P}$, Berg $\mathrm{W}$, Schneider HJ. [Stress and risk of urolith formation. I. The influence of stress on lithogenous urinary substances]. Urol Int 1981;36:199-207.

52. Brundig P, Börner RH, Schulz E, Pirlich W. Modification of the risk of urolithiasis by changes in magnesium and calcium concentrations in the urine as affected by stress conditions. $Z$ Urol Nephrol 1985;78:245-51.

53. Schmucki O, Asper R, Zortea C. [Stress and urolithiasis]. Urol Int 1984;39:159-64.

54. Walters DC. Stress as a principal cause of calcium oxalate urolithiasis. Int Urol Nephrol 1986;18:271-5.

55. Kalaitzidis RG, Damigos D, Siamopoulos KC. Environmental and stressful factors affecting the occurrence of kidney stones and the kidney colic. Int Urol Nephrol 2014;46: 1779-84.

56. Najem GR, Seebode JJ, Samady AJ, Feuerman M, Friedman L. Stressful life events and risk of symptomatic kidney stones. Int J Epidemiol 1997;26:1017-23.

57. Diniz DH, Schor N, Blay SL. Stressful life events and painful recurrent colic of renal lithiasis. J Urol 2006;176:2483-7.

58. Holmes TH, Rahe RH. The Social Readjustment Rating Scale. J Psychosom Res 1967;11:213-8.

59. Miyaoka R, Ortiz-Alvarado O, Kriedberg C, Alanee S, Chotikawanich $\mathrm{E}$, Monga M. Correlation between stress and kidney stone disease. J Endourol 2012;26:551-5.

60. Cohen S, Kamarck T, Mermelstein R. A global measure of perceived stress. J Health Soc Behav 1983;24:385-96.

61. Cohen S, Williamson G. Perceived stress in a probability sample of the United States. In S. Spacapam \& S. Oskamp (Eds.), The social psychology of health: Claremont Symposium on applied social psychology. Newbury Park 1988, CA: Sage. 